\title{
Die 31. Südtiroler Herbstgespräche in Meran
}

Prof. Dr. Wolfgang Kubelka und Prof. Dr. Sabine Glasl-Tazreiter begrüßten am 22.Oktober 2016 die über 100 Teilnehmer der traditionsreichen Veranstaltung „Phytotherapie und Phytopharmaka Praxis und Wissenschaft" mit dem diesjährigen Schwerpunkt Gynäkologie.

Bei der pharmakobotanischen Exkursion zeigten die Leiter PD Dr. Reinhard Länger und Prof. Dr. Johannes Saukel zahlreiche Pflanzen mit Bedeutung für die Phytotherapie und gaben Hintergrundinformationen. Während der ausgiebigen Wanderung kamen die Teilnehmer in zwanglose Fachgespräche.

Frau Prof. Dr. Liselotte Krenn führte in die präklinischen Grundlagen der Pflanzen in der Frauenheilkunde und deren Wirkmechanismen ein. Die präklinische Forschung unterscheidet Phytöstrogene mit Bindung an $\alpha$ - und $\beta$-Östrogenrezeptoren von SERMs, die wahrscheinlich nicht über Östrogenrezeptoren wirken. Frau Doz. DDr. Ulrike Kastner stellte bei ihrem Thema „Phytotherapie in Schwangerschaft und Stillzeit“ heraus, dass einschlägige Heilpflanzen unter entsprechender Indikationsstellung bei Unruhe und Schlafstörungen, Verdauungsstörungen, Hyperemesis gravidarum, Venenbeschwerden, Depressionen und zur Anregung des Milchflusses eingesetzt werden können. Das Heranziehen der Datenbank http://embryotox.de wurde empfohlen.

\section{Mönchspfeffer: gute Evidenz}

Die Frauenärztin Dr. Ruth Illing berichtete über traditionelle pflanzliche Arzneimittel, wobei sie das spezielle Modell nach

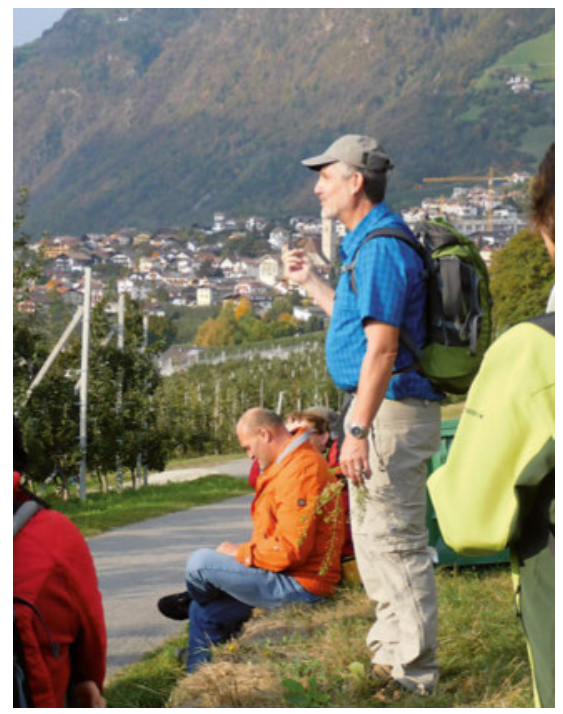

PD Dr. Reinhard Länger bei der pharmakobotanischen Exkursion oberhalb von Meran.

Jeremy Ross vorstellte, bei dem westliche Pflanzen nach den Kriterien der TCM eingeordnet und für Kombinationstees ausgewählt werden. Prof. DDr. Bernhard Uehleke zeigte auf, dass die klinische Evidenz zu Mönchspfeffer bei zyklusabhängigen Beschwerden dank überzeugender Studien bis hin zum klinischen Vergleich unterschiedlicher Dosierungen als sehr gut einzustufen ist. Hingegen zeigen mehrere hochrangig publizierte Metaanalysen zu klimakterischen Beschwerden eine ungenügende Evidenz. Im krassen Gegensatz dazu kommen iranische Experten in ihrer Metaanalyse zur Schlussfolgerung, dass eine recht umfangreiche Liste von sog. „iranischen Heilpflanzen“ als gut belegt einzustufen sei.

Prof. Dr. Karl-Heinz Wagner brachte in seinem Vortrag „Genderernährung als Baustein für Antiaging“ Beispiele für geschlechtsabhängiges Ernährungsverhalten, woraus sich Ansätze für gesundheitsrele- vantes Verhalten ableiten. Prof. Dr. Elke Heiß stellte in ihrem Vortrag „Molekulare Mechanismen von Naturprodukten - Forschungsspielerei oder Praxisrelevanz" modernste Forschungsmethoden vor, mit denen auch aus Vielstoffgemischen relevante Mechanismen erkannt werden können. Somit gefundene aktive Prinzipien können sowohl zur Verbesserung von Phytopharmaka als auch zur Leitstrukturentwicklung neuer Medikamente genutzt werden. Vorsicht sei allerdings angebracht bei der Übertragung präklinischer Ergebnisse auf die Klinik, da viele Stoffe erst verändert in die Zielgewebe gelangen.

Univ-Doz. Dr. Länger berichtete „Neues zur Risikobewertung von Arzneipflanzen“. Er beschrieb das Vorgehen mit Sicherheitsabständen bei in entsprechenden Tiermodellen auffälligen Stoffen. Aber auch alte Indikationen werden bei entsprechenden Entwicklungen revidiert.

\section{Integrative Gynäkologie im Krankenhaus Meran}

Dr. Christian Thuile führte mit einer Übersicht über die im Modell der integrativen Gynäkologie des Krankenhauses Meran die dabei verwendet Heilpflanzen auf. In dem anschließenden Workshop mit Fallpräsentationen wurden interaktiv mit den Teilnehmern phytotherapeutische Konzepte vorgestellt und diskutiert.

Alle Beiträge wurden recht kritisch und teilweise sehr kontrovers diskutiert. Es ist bemerkenswert, dass es dank des Engagements der Veranstalter noch solche Veranstaltungen in einer solch schönen Atmosphäre gibt.

Dr. Dr. Bernhard Uehleke, Berlin 